\title{
Expanded hammerhead ribozymes containing addressable three-way junctions
}

\author{
MARKUS WIELAND, MANUELA GFELL, and JÖRG S. HARTIG \\ Department of Chemistry and Konstanz Research School of Chemical Biology (KoRS-CB), University of Konstanz, D-78457 Konstanz, Germany
}

\begin{abstract}
Recently, hammerhead ribozyme (HHR) motifs have been utilized as powerful tools for gene regulation. Here we present a novel design of expanded full-length HHRs that allows attaching additional functionalities to the ribozyme. These features allowed us to construct a very efficient artificial riboswitch in bacteria. Following the design of naturally occurring three-way junctions we attached an additional helix (IV) to stem I of the HHR while maintaining very fast cleavage rates. We found that the cleavage activity strongly depends on the exact design of the junction site. Incorporation of the novel ribozyme scaffold into a bacterial mRNA allowed the control of gene expression mediated by autocatalytic cleavage of the ribozyme. Appending an aptamer to the newly introduced stem enabled the identification of very powerful theophylline-inducible RNA switches by in vivo screening. Further investigations revealed a cascading system operating beyond the ribozyme-dependent mechanism. In conclusion, we extended the hammerhead toolbox for synthetic biology applications by providing an additional position for the attachment of regulatory modules for in vivo control of gene expression.
\end{abstract}

Keywords: hammerhead ribozyme; regulation of gene expression

\section{INTRODUCTION}

Nature provides a variety of RNA elements located in the $5^{\prime}$-untranslated region (5'-UTR) of messenger RNAs acting as regulators of gene expression without the need for involved protein factors (Winkler and Breaker 2005). In addition to the discovery of naturally occurring RNA-only mechanisms for controlling gene expression, several examples of artificial RNA-based switches have been constructed (Win and Smolke 2007b; Suess and Weigand 2008; Wieland and Hartig 2008). Specific binding of metabolites to such riboswitches triggers a structural change affecting transcriptional or translational efficacy. An exception represents the $g l m S$ riboswitch, since binding of its metabolite glucosamine-6-phosphate results in autocatalytic cleavage of the $g l m S$ mRNA, followed by degradation mediated by a specific nuclease (Winkler et al. 2004; Collins et al. 2007). Long before this mechanism was discovered, self-cleaving RNAs have been studied in great detail (Carola and Eckstein 1999; Cech 2002; Fedor and Westhof 2002).

Reprint requests to: Jörg S. Hartig, Department of Chemistry and Konstanz Research School of Chemical Biology (KoRS-CB), University of Konstanz, Universitätsstrasse 10, D-78457 Konstanz, Germany; e-mail: joerg.hartig@uni-konstanz.de; fax: 49-7531-885140.

Article published online ahead of print. Article and publication date are at http://www.rnajournal.org/cgi/doi/10.1261/rna.1220309.
Among these, the hammerhead ribozyme (HHR), originally found in viroids, catalyzes a magnesium-dependent phosphodiesterase reaction leading to site-specific selfcleavage (Blount and Uhlenbeck 2002). First structural findings suggested a hammerhead-like structure comprised of three helical stems flanking a catalytic core (Forster and Symons 1987). While the core sequence is conserved, the flanking helices, termed stems I, II, and III, can be varied. The HHR motif is capable of catalyzing RNA cleavage in trans if divided into a catalytic and a substrate strand (Uhlenbeck 1987). Recent data by Scott and coworkers delivered new insights into structural requirements of extended HHR motifs: Tertiary contacts between stem I and II distant from the catalytic core stabilize the active conformation of the HHR (Martick and Scott 2006). This leads to an increased cleavage activity compared to minimal HHR motifs lacking the tertiary interactions, and enables ribozyme cleavage to occur at low magnesium concentrations as found inside cells (Khvorova et al. 2003; Canny et al. 2004).

Pioneering the application of ribozymes for in cis control of gene expression, Mulligan and coworkers inserted a fulllength HHR into the mRNA of a eukaryotic reporter gene. Self-cleavage resulted in a destabilized mRNA, eventually inhibiting gene expression (Yen et al. 2004). Reconstitution of gene expression was achieved by the addition of 
nucleoside analogs. Upon unspecific incorporation into the cellular RNA, the analogs interfere with ribozyme activity, resulting in full-length mRNA and restored gene expression levels (Yen et al. 2004, 2006). However, the mechanism of this ribozyme switch is rather unspecific, potentially resulting in toxic side effects of the nucleoside analogs.

In order to obtain a specific HHR/ligand interaction, aptamers can be introduced into the ribozyme. Aptamers are short RNA sequences specifically binding their respective ligands with high affinity (Jenison et al. 1994). Several examples of so-called aptazymes based on minimal-motif HHRs (Soukup and Breaker 1999b; Jose et al. 2001; Piganeau et al. 2001a,b; Hartig et al. 2002) as well as on other ribozymes (Amontov and Jaschke 2006) have been described in vitro. Ligand binding to the aptamer domain triggers a change of ribozyme activity (Tang and Breaker 1998). The connection site of ribozyme and aptamer, the so-called communication module, is often generated by in vitro selection protocols (Soukup and Breaker 1999a). Recently, Smolke and coworkers attached either theophylline (Jenison et al. 1994) or tetracycline aptamers (Berens et al. 2001) to stem I or II of a full-length HHR in the $3^{\prime}$ UTR of a reporter gene in yeast (Win and Smolke 2007a). The aptazymes generated by rational design showed liganddependent control of gene expression, likely mediated by switching the tertiary interactions between stems I and II (Win and Smolke 2007a).

Since the genetic mechanisms differ strongly in bacteria and yeast, a simple transfer of the yeast RNA switches to function in bacteria is not sufficient. The eukaryotic ribozyme switches discussed above likely act via cleaving stabilizing elements such as the $5^{\prime}$-capped end or the $3^{\prime}$ polyA tail in order to enhance degradation of the message. In bacteria, a different approach has to be realized. The ribosome binding site (RBS), which needs to be single stranded for efficient translation initiation, offers a worthwhile target. Recently, we demonstrated that bacterial gene expression can be controlled by an HHR if the RBS is incorporated into stem I of the hammerhead ribozyme (Wieland and Hartig 2008). Self-cleavage of the HHR results in liberation of the RBS and enables efficient translation initiation. Furthermore, by attaching a theophylline aptamer to stem III followed by an in vivo screening for an optimized connecting sequence, we identified an artificial riboswitch based on a theophylline-dependent aptazyme (theoHHAz) (Wieland and Hartig 2008). In a second study, we utilized a similar hammerhead ribozyme setup in order to attach naturally occurring, riboswitch aptamer domains. The approach enabled us to identify both artificial switches getting inhibited as well as activated by addition of thiamine to the growth medium (Wieland et al. 2009). In a different setup, minimal theophyllinedependent ribozymes enabled switching of gene expression under certain growth conditions (Ogawa and Maeda 2008). In the present study we aim at expanding the hammerhead scaffold in order to introduce further positions for the connection of the ribozyme to an mRNAs as well as for tuning ribozyme activity. Although it was shown that all three stems can, in principle, be used for the latter task (Win and Smolke 2007a; Wieland and Hartig 2008), we wondered if there are other positions an aptamer can be introduced at. The possibility to control a ribozyme simultaneously at multiple sites would enable the development of logic gates, accepting more than one ligand as input. In vitro experiments performed with trans ribozymes suggested stem I to be a suitable choice. An HHR containing a nicked strand in between the catalytic core and the stem I/stem II tertiary interaction site of stem I still shows fast cleavage rates at $0.5 \mathrm{mM} \mathrm{Mg}^{2+}$ (see Fig. 1A; Burke and Greathouse 2005). Interestingly, Weinberg and Rossi reported that attaching an additional stem (termed stem IV) to this discontinuity (see Fig. 1B) severely reduces cleavage activity and the modified HHR seems to be incapable of cleavage at submillimolar $\mathrm{Mg}^{2+}$ concentrations (Weinberg and Rossi 2005).

By attaching an additional stem IV to stem I of the hammerhead ribozyme, we aimed at enlarging the ribozyme scaffold by an artificial three-way junction. In order to implement such a branching point into the ribozyme, we adapted its design to the architecture of naturally occurring three-way junctions (Lescoute and Westhof 2006). The investigation of the structural requirements of natural RNA three-way junctions turned out to be crucial, and enabled us to construct an enhanced HHR scaffold without disturbing the formation of the tertiary interactions (see Fig. 1C). Due to its very fast cleavage kinetics even at $0.2 \mathrm{mM}$ $\mathrm{Mg}^{2+}$, this enhanced HHR can also modulate bacterial gene expression if placed in the RBS-liberating setup as described above. Furthermore, by in vivo screening we were able to identify a very powerful theophylline-dependent artificial switch of gene expression based on the expanded HHR scaffold.
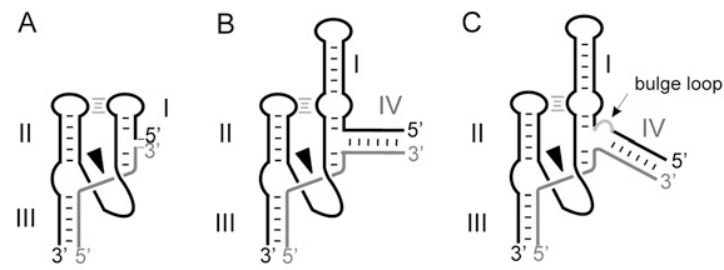

FIGURE 1. Construction of an expanded HHR scaffold. Naturally occurring HHR motifs comprise three helices. Tertiary interactions (depicted as gray lines) between stem I and II enable very fast cleavage kinetics even at low intracellular $\mathrm{Mg}^{2+}$ concentrations (Martick and Scott 2006). (A) A nicked strand in stem I does not interfere with fast cleavage properties of the HHR (Burke and Greathouse 2005). (B) Attaching a fourth stem at this position results in severely reduced cleavage activity (Weinberg and Rossi 2005). (C) Our design of expanded hammerhead ribozymes is based on a short bulge loop sequence enabling rapid cleavage at low $\mathrm{Mg}^{2+}$ concentrations. 


\section{RESULTS}

\section{Design of an expanded HHR scaffold in vitro}

In order to expand the repertoire of the HHR scaffold, we attached an additional stem IV to stem I in between the catalytic core and the tertiary interaction site of stems I and II, see Fig. 2A). The resulting structure (three-way HHR) comprises a new three-way junction consisting of stem IV and the discontinued stem I. As can be found in the literature, immediate attachment of an additional stem results in significant loss of activity (Weinberg and Rossi 2005). In order to retain catalytic activity of the ribozyme, we attempted to design the three-way junction in a way that allows coaxial stacking of both sides of helix I. For this purpose, we surveyed known structures of naturally occurring three-way junctions: In most classes, the three helices forming a three-way junction are connected by nucleotides not involved in canonical pairs, forming bulge loops with varying lengths (Lescoute and
Westhof 2006). Depending on the bulge loop lengths and the arrangement of the stems, three-way junctions have been classified into three families (Lescoute and Westhof 2006). In order to construct the artificial three-way junction needed for the expanded scaffold, we decided to insert a bulge loop at the junction site (see Fig. 2A). This design represents a family C member in the categories introduced by Westhof and coworkers. Comparing various three-way junctions, length, and sequence of the bulge loops differ strongly (Lescoute and Westhof 2006). Thus, we examined the influence of the bulge loop length on the activity of our three-way HHRs. Initially, we synthesized ribozymes containing bulge loop sequences composed of $5^{\prime}-\mathrm{A}(\mathrm{U})_{n}-3^{\prime}$ (with $n=0-3$ ), since mfold secondary structure prediction (Zuker 2003) resulted in the designated ribozyme fold.

For the initial in vitro experiments, single turnover kinetics of in trans cleaving three-way HHRs were assayed (see Fig. 2A). Upon immediate attachment of stem IV to the ribozyme lacking any noncanonical nucleotides in the

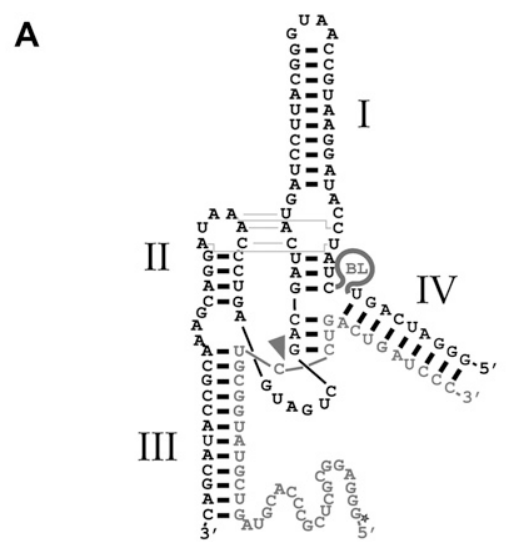

C

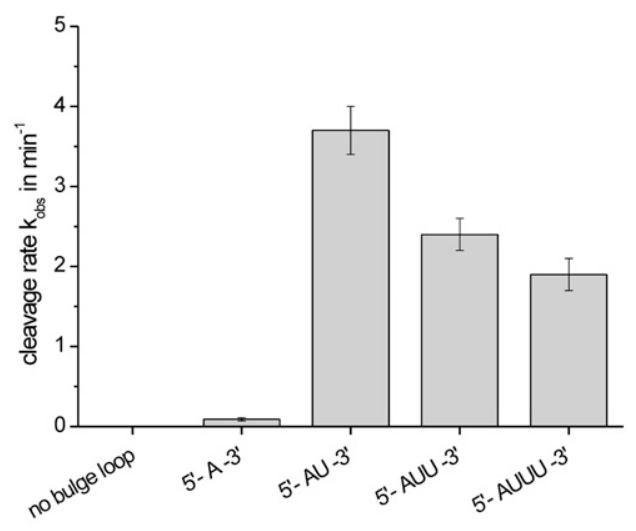

B

D
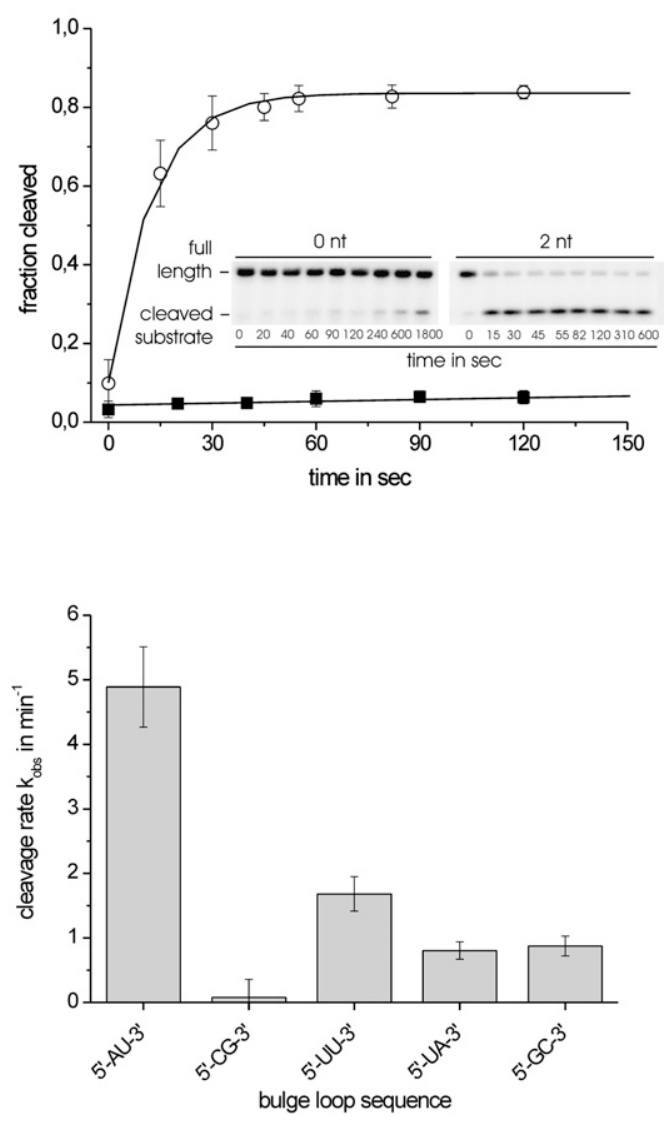

FIGURE 2. In trans, single turnover cleavage kinetics of purified three-way HHRs in dependence of the bulge loop length. (A) Design of fastcleaving three-way HHRs. Arrow indicates cleaving site; black characters: ribozyme strand; gray characters: $5^{\prime}$-labeled substrate strand; gray lines: tertiary interactions between stem I and II after (Martick and Scott 2006); bulge loop sequence position is indicated as BL. (B) Cleavage kinetic and example of corresponding PAGE gel of three-way HHR without bulge loop (black squares) and with the 2-nt sequence AU (open circles). $(C, D)$ In trans, single turnover cleavage rates $\left(k_{\mathrm{obs}}\right)$ of three-way HHRs with $(C)$ increasing bulge loop lengths and $(D)$ different bulge loop sequences in the presence of $0.2 \mathrm{mM} \mathrm{Mg}^{2+}$ at $37^{\circ} \mathrm{C}$. 
three-way junction, only very little ribozyme activity could be observed at $0.2 \mathrm{mM} \mathrm{Mg}^{2+}$ (see Fig. 2B). The introduction of a single adenosine in the junction results in a cleavage rate $k_{\mathrm{obs}}$ of $0.19 \mathrm{~min}^{-1}$ at $0.2 \mathrm{mM} \mathrm{Mg}^{2+}$. Upon addition of a second nucleotide, the cleavage rate increased remarkably to $4.9 \mathrm{~min}^{-1}$ (see the sequence AU). Inserting more nucleotides did not show improvement but reduced the cleavage rates slightly (see Fig. 2C). Interestingly, at 0.2 $\mathrm{mM} \mathrm{Mg}^{2+}$ the addition of 2 nucleotides (nt) at the junction site results in dramatically activated ribozymes. Catalytic activity of the inactive variant lacking noncanonical nucleotides can be restored by increasing the magnesium concentration. For detailed kinetics determined for the ribozymes at $0.2,0.5,1$, and $10 \mathrm{mM} \mathrm{Mg}^{2+}$ concentrations see Table 1 . In order to further characterize the junction site requirements, we examined several sequences of the best-performing bulge loop size with $2 \mathrm{nt}$ in length. The cleavage rate was highest in the case of the already tested $5^{\prime}$-AU-3' bulge loop, but was reduced in all other tested examples (see Fig. 2D). In conclusion, the bulge loop sequence $5^{\prime}$-AU-3' represents the fastest three-way HHR tested in vitro, and is still highly active at magnesium concentrations as low as $200 \mu \mathrm{M}$.

TABLE 1. In trans, single turnover-cleavage rates ( $k_{\text {obs }}$ ) of three-way HHRs with increasing bulge loop lengths assayed at various $\mathrm{Mg}^{2+}$ concentrations

\begin{tabular}{|c|c|c|c|c|c|c|c|}
\hline \multirow{2}{*}{$\begin{array}{l}\text { Bulge } \\
\text { loop } \\
\text { size } \\
0 \mathrm{nt}\end{array}$} & \multirow{2}{*}{$\begin{array}{c}\mathrm{Mg}^{2+} \\
(\mathrm{mM})\end{array}$} & \multicolumn{2}{|c|}{$\begin{array}{c}k_{\mathrm{obs}} \\
\left(\mathrm{min}^{-1}\right)\end{array}$} & \multicolumn{2}{|r|}{$F_{\infty}$} & \multicolumn{2}{|r|}{$F_{0}$} \\
\hline & & 8.4 & \pm 1.1 & 0.92 & \pm 0.00 & 0.02 & \pm 0.02 \\
\hline & 1 & 1.1 & \pm 0.1 & 0.90 & \pm 0.01 & 0.03 & \pm 0.02 \\
\hline & 0.5 & 0.3 & \pm 0.0 & 0.84 & \pm 0.01 & 0.02 & \pm 0.01 \\
\hline & 0.2 & $<0.04$ & & & & & \\
\hline \multirow[t]{4}{*}{$1 \mathrm{nt}$} & 1 & 5.8 & \pm 1.0 & 0.90 & \pm 0.02 & 0.02 & \pm 0.04 \\
\hline & 0.5 & 2.6 & \pm 0.3 & 0.85 & \pm 0.02 & 0.03 & \pm 0.04 \\
\hline & 0.2 & 0.1 & \pm 0.1 & 0.86 & \pm 0.07 & 0.01 & \pm 0.02 \\
\hline & 0.1 & $<0.04$ & & & & & \\
\hline \multirow[t]{4}{*}{$2 \mathrm{nt}$} & 1 & 8.0 & \pm 1.0 & 0.98 & \pm 0.01 & 0.03 & \pm 0.02 \\
\hline & 0.5 & 7.2 & \pm 1.0 & 0.96 & \pm 0.01 & 0.04 & \pm 0.02 \\
\hline & 0.2 & 3.7 & \pm 0.3 & 0.89 & \pm 0.01 & 0.02 & \pm 0.02 \\
\hline & 0.1 & 0.1 & \pm 0.0 & 0.63 & \pm 0.03 & 0.05 & \pm 0.01 \\
\hline \multirow[t]{4}{*}{$3 \mathrm{nt}$} & 1 & 8.6 & \pm 1.5 & 0.95 & \pm 0.01 & 0.01 & \pm 0.02 \\
\hline & 0.5 & 7.2 & \pm 1.0 & 0.94 & \pm 0.01 & 0.01 & \pm 0.02 \\
\hline & 0.2 & 2.4 & \pm 0.2 & 0.88 & \pm 0.01 & 0.01 & \pm 0.02 \\
\hline & 0.1 & $<0.04$ & & & & & \\
\hline \multirow[t]{4}{*}{$4 \mathrm{nt}$} & 1 & 7.6 & \pm 1.6 & 0.93 & \pm 0.01 & 0.03 & \pm 0.04 \\
\hline & 0.5 & 7.2 & \pm 1.4 & 0.91 & \pm 0.01 & 0.01 & \pm 0.04 \\
\hline & 0.2 & 1.9 & \pm 0.2 & 0.83 & \pm 0.02 & 0.04 & \pm 0.04 \\
\hline & 0.1 & 0.1 & \pm 0.0 & 0.71 & \pm 0.06 & 0.07 & \pm 0.02 \\
\hline \multirow[t]{4}{*}{$5 \mathrm{nt}$} & 1 & 7.4 & \pm 1.4 & 0.93 & \pm 0.01 & 0 & \pm 0.03 \\
\hline & 0.5 & 7.1 & \pm 1.3 & 0.89 & \pm 0.01 & 0.01 & \pm 0.03 \\
\hline & 0.2 & 1.0 & \pm 0.1 & 0.78 & \pm 0.02 & 0.03 & \pm 0.03 \\
\hline & 0.1 & $<0.04$ & & & & & \\
\hline
\end{tabular}

$F_{\infty}$ : Maximal cleaved fraction (single turnover reactions with $10 \times$ substrate excess); $F_{0}$ : cleaved fraction prior to $\mathrm{Mg}^{2+}$ addition.

\section{Cleavage activity of three-way HHR in bacteria}

Recently, we constructed an artificial RNA switch in Escherichia coli based on a full-length in cis cleaving HHR. In this setup, the RBS of the reporter gene eGFP is incorporated into an extended stem I of the HHR; see Figure 3A. If the HHR is inactive, the RBS is blocked, and hence, translation is inhibited. An active HHR, however, liberates the RBS by self-cleavage and translation is initiated (Wieland and Hartig 2008). This setup was used in order to test whether the designed three-way HHRs act as modulators of gene expression in vivo. As a control, the threeway HHR was inactivated by a point mutation in the active core. While eGFP is still expressed in a clone harboring an active three-way HHR in the reporter mRNA, expression can be inhibited almost completely by inactivating the three-way HHR by the point mutation. However, the absolute expression level of the three-way HHR clone is reduced compared to the unmodified HHR clone; see Figure 3D. In analogy to the previous in vitro experiments, the dependence of gene expression on the size of the bulge loop at the three-way junction site was examined in the in vivo setup. In accordance to the data obtained in vitro, omitting the bulge loop reduced eGFP expression to the same level as the variant inactivated by the point mutation in the catalytic core (see Fig. 3E), demonstrating that additional nucleotides at the junction site are necessary for ribozyme activity in vivo. Best results are obtained with a bulge loop length of $3 \mathrm{nt}$, whereas constructs harboring $2 \mathrm{nt}$, as well as $4 \mathrm{nt}$, still enabled efficient eGFP expression. If the bulge loop consists of only $1 \mathrm{nt}$, gene expression is significantly weakened. In conclusion, the in vivo activities of the different three-way HHRs as judged by their modulation of gene expression are in good accordance with the observed cleavage rates of the isolated ribozymes as determined before.

\section{Construction of a theophylline-dependent three-way HHR}

Since eGFP expression of the three-way HHR-containing clones is directly dependent on the size of the bulge loop at the junction site, we reasoned that it should be possible to utilize a structural rearrangement at this site for controlling the activity of the ribozyme. Ligand-induced rearrangements such as strand slippage have been reported in the literature before (Soukup and Breaker 1999b). For a general discussion of RNA structural rearrangements, see Micura and Hobartner (2003) and Furtig et al. (2007). The identification of sequences allowing the ligand-dependent switching of the bulge loop size would represent a skillful way of controlling the HHR activity and hence gene expression. As proof of principle, we attached a theophylline aptamer to stem IV of the three-way HHR (based on the variant with a bulge loop size of $3 \mathrm{nt}$ ) in order to design a theophylline-dependent three-way aptazyme (three-way 
A

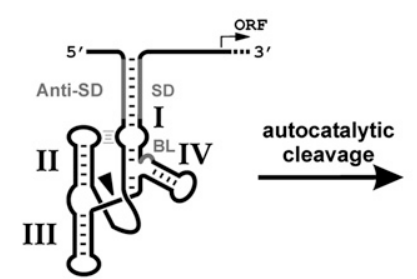

inactive 3way HHR gene expression OFF

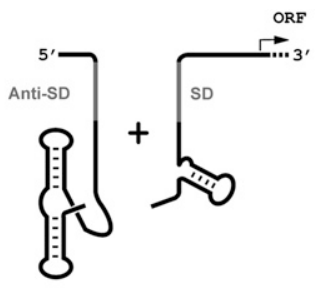

cleaved 3way HHR gene expression ON
B

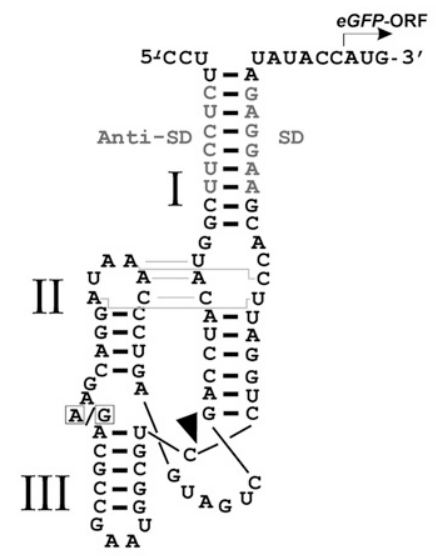

D

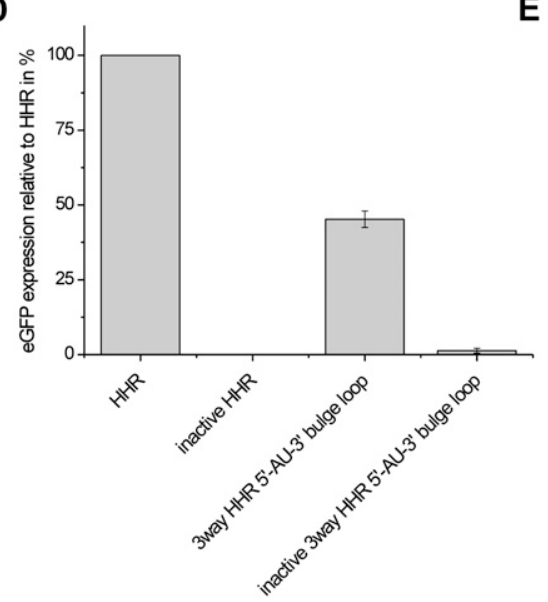

C

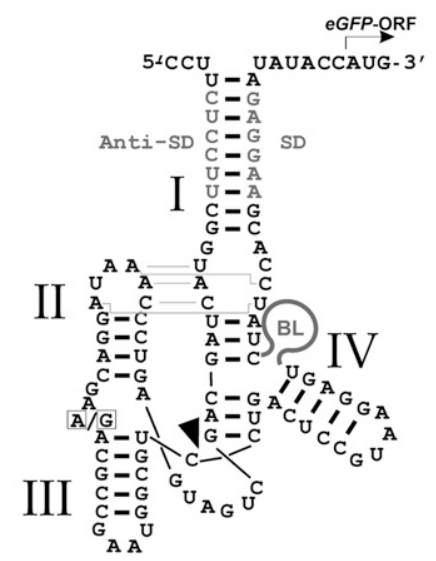

$E$

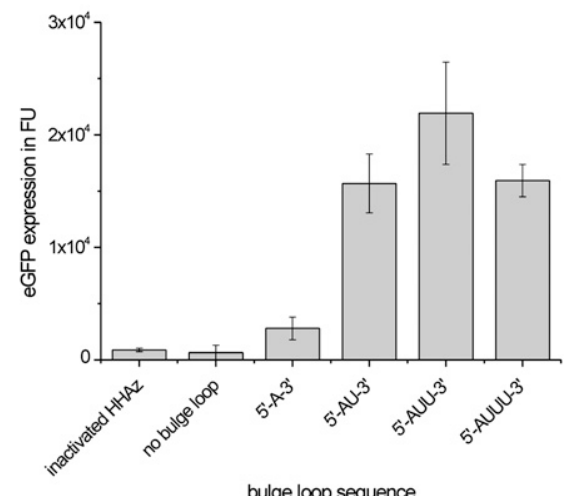

FIGURE 3. Three-way HHRs introduced into the 5'-UTR of bacterial reporter gene modulates gene expression. (A) Mechanism of HHR-mediated eGFP expression. Inactivated three-way HHR results in a blocked SD sequence. If an active three-way HHR cleaves the mRNA, eGFP expression is restored. $(B, C)$ Design of an artificial riboswitch based on $(B)$ the HHR or $(C)$ the three-way HHR. Nucleotides mutated to inactivate ribozymes are boxed (A to G). Tertiary interactions are depicted as gray lines corresponding to Martick and Scott (2006). $(D)$ Gene expression levels of variants relative to the wild-type plasmid lacking a ribozyme. Inactivated ribozymes inhibit gene expression by blocking the Shine-Dalgarno (SD) sequence (gray). (E) eGFP expression of clones with inserted three-way HHRs in dependence of different bulge loop lengths.

theoHHAz). A suitable connecting sequence was obtained by in vivo screening. For this purpose, $5 \mathrm{nt}$ of the connection site between the HHR and aptamer at the three-way junction site were randomized (see boxed nucleotides in Fig. 4A). After library construction in E. coli, eGFP expression levels were screened in the presence and absence of $2 \mathrm{mM}$ theophylline. After screening of 2000 individual clones, we

identified a sequence (UUCCG) which exhibited a highly theophylline-dependent eGFP expression; see Figure 4A. Reporter gene expression of the clone threeway theoHHAz in the absence of theophylline is barely detectable, but can be significantly induced upon the addition of $2 \mathrm{mM}$ theophylline; see Figure $4 \mathrm{~B}$ and C. Inactivating point mutants of the three-way theoHHAz demonstrated that the switching mechanism is dependent on the ribozyme activity since gene expression is absent in all inactivated point mutants irrespective of the absence or presence of theophylline; see Figure 4B. Importantly, the expression level of the induced state corresponds to the expression of the nonregulated mother clone three-way HHR, thereby displaying a maximum switching range from barely detectable to full gene expression; see Figure 4C. Addition of the theophylline analog caffeine, which differs from theophylline by a single methylgroup, showed no effect on eGFP expression of three-way theoHHAz, confirming that the effects are indeed mediated by aptamer-based recognition of the respective ligand (see Fig. 4D). Neither theophylline nor caffeine was able to induce the parental, nonregulated, three-way HHR variant, but just slightly inhibited gene expression at higher concentrations.

\section{Switching mechanism of theophylline-dependent three-way theoHHAz}

Subsequently, the underlying mechanism of gene regulation by the identified clone was examined. Ribozyme assays with isolated in cis three-way theoHHAz were performed to investigate the theophylline dependence of the cleavage rate. For this purpose, in vitro transcribed threeway HHR and three-way theoHHAz were assayed in the absence and presence of $2 \mathrm{mM}$ theophylline and caffeine at 0.2 $\mathrm{mM} \mathrm{Mg}^{2+}$. Whereas the ribozyme activity of the three-way HHR is unaffected by either theophylline or caffeine, the cleavage rate of the three-way theoHHAz is induced twofold in the presence of $2 \mathrm{mM}$ theophylline but remains unaffected by caffeine (see Fig. 5B). The results are in accordance with the data obtained in vivo and evidence that the identified three-way HHR is a theophylline-dependent aptazyme. 


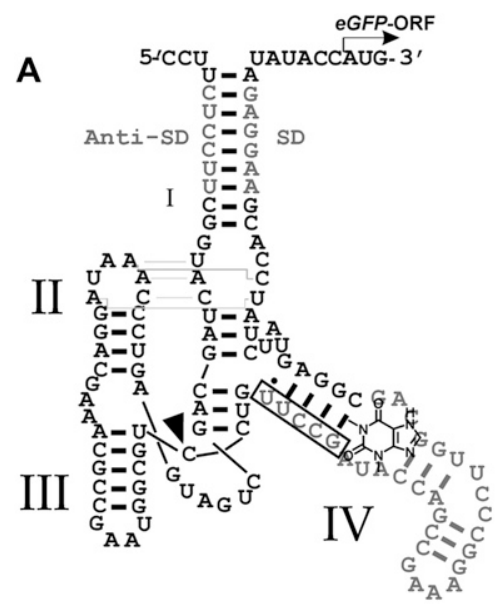

B
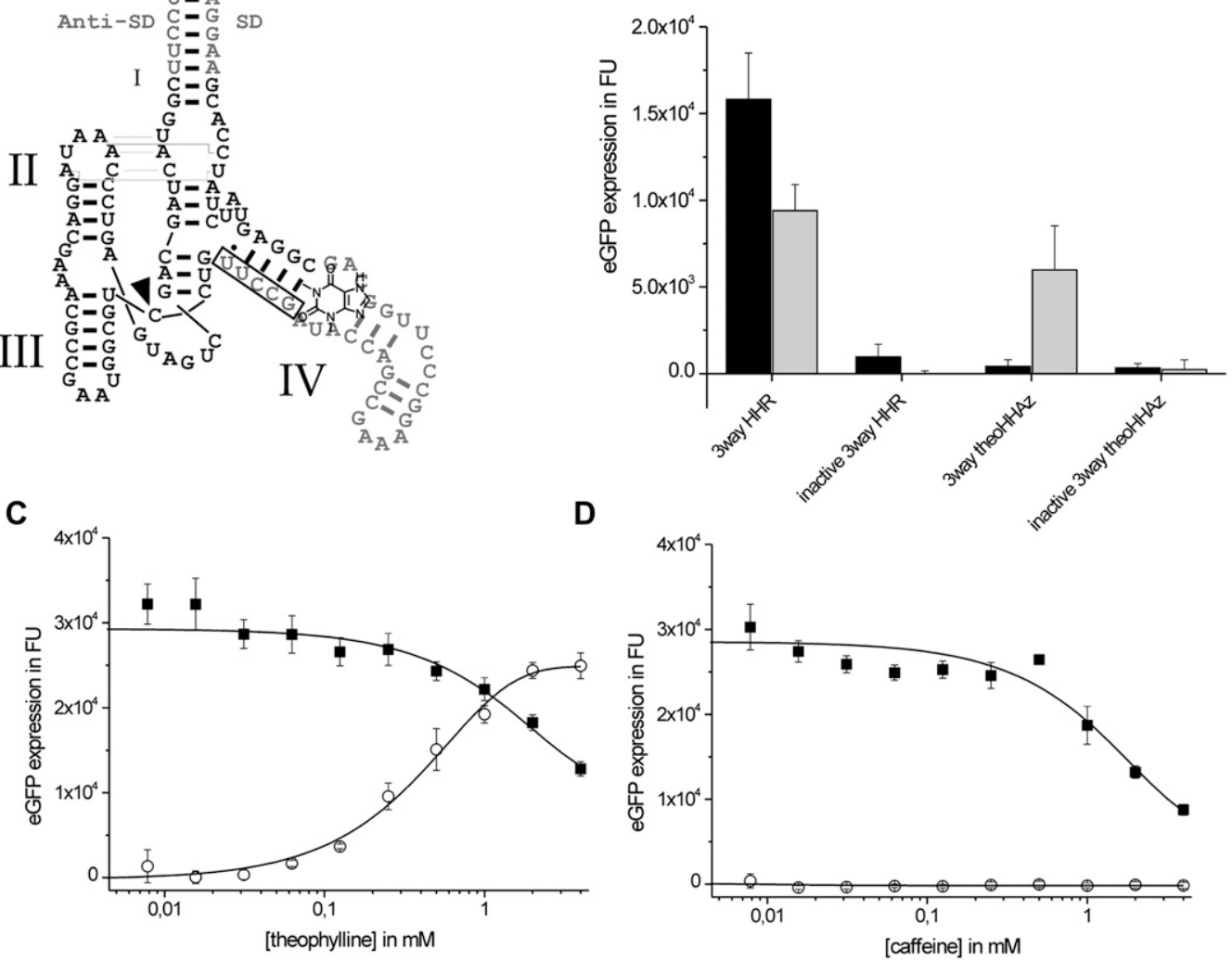

FIGURE 4. Construction of a three-way HHR-based RNA switch of gene expression by in vivo screening. (A) A theophylline aptamer (gray) was attached to stem IV. In order to identify a suited communication module, boxed nucleotides were randomized. After in vivo screening, the shown sequence was identified as best theophylline-inducible clone. (B) Loss-of-function mutations of ribozymes: Point mutations in the catalytic core render the ribozymes inactive. In vivo gene expression is absent in case of the inactivating mutants irrespective of the absence (black columns) or presence (gray bars) of $2 \mathrm{mM}$ theophylline. $(C, D)$ Dependence of gene expression on $(C)$ theophylline and $(D)$ caffeine. Reporter gene expression of identified clone (open circles) can be induced by theophylline in a dose-dependent manner, whereas the theophylline analog caffeine has no effect. The parental, nonregulated, three-way HHR lacking the aptamer (black squares) did not show theophylline-dependent induction but gets slightly inhibited at higher concentrations by both theophylline and caffeine.

It is, however, unlikely that a 100 -fold induction of gene expression is exclusively based on a twofold induction of the cleavage rate. In contrast to the aptazyme design employed in our previous study (Wieland and Hartig 2008), in the present three-way junction setup the aptamer remains at the $5^{\prime}$-end of the mRNA after ribozyme cleavage has occurred. Hence, it is conceivable that a ribozyme-independent mechanism is also involved in the regulatory process. In order to further characterize the mechanism, theophyllinemediated control of gene expression in vivo that takes place after ribozyme cleavage was surveyed by introducing only the $3^{\prime}$-cleavage product of the three-way theoHHAz ("cleaved fragment") aptazyme into the 5 '-UTR of the $e G F P$ mRNA instead of the complete ribozyme; see Supplementary Figure S1. We found that the reporter gene expression of this clone harboring only the aptamer is theophyllinedependent as well. In order to shed light on the underlying mechanism, we determined mRNA levels of the correspond- ing mRNAs. Ligand-dependent transcriptional efficiency and mRNA stability should result in diferences in mRNA levels, whereas interference with ribosomal initiation would not result in changed mRNA levels. Following mRNA extraction and RTPCR we found the same mRNA abundances in the absence and presence of theophylline for all studied clones, excluding the possibility of theophylline-dependent changes in mRNA levels (data not shown).

\section{DISCUSSION}

The HHR has been shown to be a powerful and versatile tool for the in cis regulation of gene expression (Yen et al. 2004, 2006; Win and Smolke 2007a; Wieland and Hartig 2008). In order to broaden its applicability, additional ways of connecting the hammerhead to natural RNAs are highly warranted. We successfully introduced a fourth helix to the natural HHR scaffold while retaining its fast-cleaving abilities. 
A

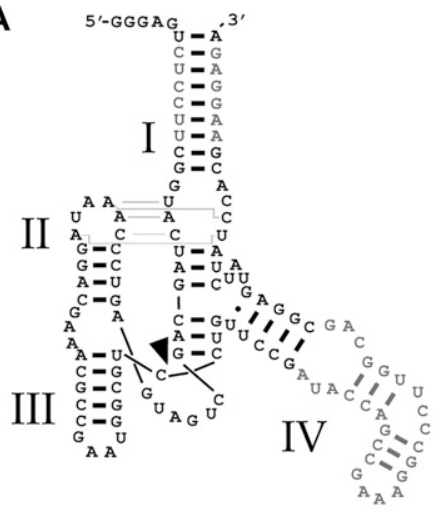

B

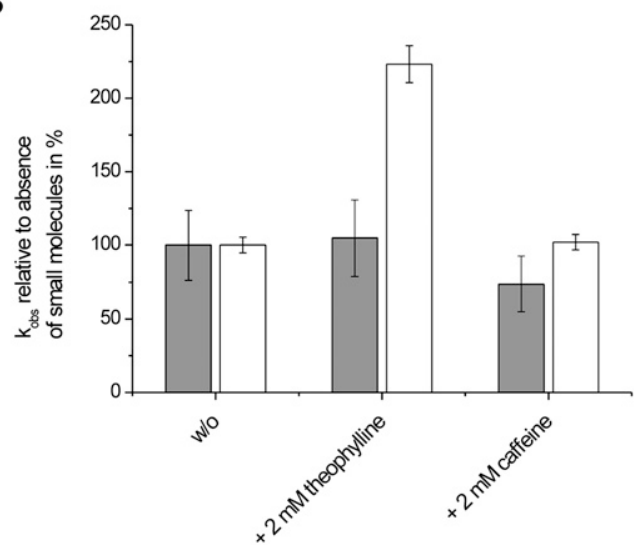

FIGURE 5. In vitro cleavage assay of identified three-way theoHHAz ribozyme. $(A)$ Design of the in cis three-way theoHHAz. (B) Relative cleavage rates of the three-way HHR (gray) lacking ligand dependency and the three-way theoHHAz (white) in the presence and absence of either $2 \mathrm{mM}$ theophylline or caffeine. Only three-way theoHHAz is induced more than twofold upon addition of theophylline.

The expanded three-way HHR is capable of modulating gene expression in vivo using our setup of ribozyme-mediated masking of the RBS. It has been shown previously that one can introduce a discontinuity in stem I of the full-length HHR and still observe cleavage at submillimolar magnesium concentrations (Burke and Greathouse 2005). The reduced cleavage activity observed with this nicked HHR could be explained by a weakened stability of stem I. Interestingly, elongating the ends of the nicked strands with a new helix does not increase cleavage rate but results in an impaired ribozyme displaying only slow cleavage at high $(10 \mathrm{mM}) \mathrm{Mg}^{2+}$-concentrations (Weinberg and Rossi 2005). Possible explanations for this behavior upon introduction of the new stem structure could be either further destabilization of stem I or disturbed tertiary interactions of stems I and II.

In order to engineer a hammerhead ribozyme that contains a fourth helix, a three-way junction has to be inserted into the ribozyme scaffold. Since several structures of naturally occurring three-way junctions are solved, we considered common features that need to be present for the successful construction of an artificial three way junction. Notably, in all natural three-way junctions the stems are not simply attached to each other but harbor noncanonical nucleotides at the junction site (Lescoute and Westhof 2006). Inspired by the natural design, we constructed an artificial three-way junction in stem I of the fulllength HHR (three-way HHR) and introduced additional nucleotides at the junction. Interestingly, in vitro cleavage assays of our designed three-way HHRs demonstrated a strong dependence of the cleavage rate on the number of nucleotides introduced at the junction site. In addition, the sequence of the inserted nucleotides affects three-way HHR activity. Replacing $5^{\prime}$-AU-3' by other dinucleotides leads to clearly reduced cleavage rates in our examples. Mfold structure predictions of these variants suggest that these

nucleotides enable the formation of competing, inactive structures. In addition to the successful construction of an expanded HHR scaffold cleaving with high activity, we implemented the new design in our previously introduced setup for the modulation of gene expression in bacteria (Wieland and Hartig 2008). Investigation of an inactivating point mutant enabled the conclusion that the three-way HHR is cleaving in vivo, resulting in activation of gene expression. The influence of the bulge loop size on the expression levels in vivo are in good accordance with the data obtained for the isolated ribozymes.

The design of the three-way junction ribozymes and the confirmation that the junction architecture is determining the in vivo ribozyme activity set the basis to develop a novel ligand-dependent regulator of gene expression. By attaching an aptamer to the newly introduced helix IV followed by in vivo screening for the optimum connection sequence, we were able to identify a very powerful switch of gene expression. While cleavage rates of in vitro synthesized three-way HHR was independent of theophylline, the aptamer-containing clone optimized by an in vivo screening experiment (three-way theoHHAz) showed an induction of ribozyme activity at $0.2 \mathrm{mM} \mathrm{Mg}^{2+}$. The identified three-way theoHHAz displayed cleavage rates of $1.5 \mathrm{~min}^{-1}$ and $3.4 \mathrm{~min}^{-1}$ at $0.2 \mathrm{mM} \mathrm{Mg}^{2+}$ for the two respective states of the ribozyme in vitro. These rates are in the same range as our previously reported stem III-based theophylline-dependent aptazymes (Wieland and Hartig 2008; Wieland et al. 2009). This observation points at the possibility that the identified rates reside in an optimal window of cleavage activities of hammerhead ribozymes for the modulation of gene expression in mRNAs of $E$. coli. Upon insertion of the $3^{\prime}$-cleavage product into the $e G F P$ mRNA, dose-dependent regulation of expression upon addition of theophylline was still observed. Nevertheless, in the ribozyme-containing constructs, the switching properties rely on the catalytic activity of the ribozyme. The finding could be explained by a potential cascading mechanism consisting of a first, theophylline dependent aptazyme cleavage reaction and a second, as well theophyllinedependent structural rearrangement of the $5^{\prime}$-UTR; see Supplemental Figure S2. The latter mechanism resembles the RNA switches constructed by Gallivan and coworkers (Lynch et al. 2007), although the architecture differs by the distance between the SD and the theophylline aptamer.

By attaching an additional stem IV to stem I using optimized three-way junctions, we have successfully expanded the hammerhead scaffold, offering a further attachment site for functional extensions of the autocatalytic 
motif. We established an additional means of using hammerhead ribozymes as artificial switches of gene expression in bacteria. In general, we attempt to regulate nucleic acid functions by augmenting the respective motifs with modules that allow control via external stimuli. In order to do so, the development of a modular toolbox is especially important. Here, we have shown that a very compact, small RNA motif such as the hammerhead ribozyme can be engineered to carry additional structural features. Furthermore, these additional components can be employed for switching the given activity by incorporating aptamer sequences. With the rapidly increasing number of attachment sites for regulatory motifs in the hammerhead scaffold (all three naturally occurring stems have been used for aptamer attachment, here we introduced a fourth possibility), researchers should be soon able to engineer logic gates that accept multiple inputs based on the hammerhead ribozyme. In addition, the present study highlights the importance of consulting details of naturally occurring RNA motifs when engineering artificial RNA functions for synthetic biology applications.

\section{MATERIAL AND METHODS}

\section{Determination of ribozyme cleavage rates}

All in vitro experiments were performed using $\mathrm{ddH}_{2} \mathrm{O}$ instead of Millipore $\mathrm{H}_{2} \mathrm{O}$ to guarantee the complete absence of $\mathrm{Mg}^{2+}$.

\section{In trans $\mathrm{HHR}$ activities}

Synthetic DNA templates of the HHRs were PCR amplified and subsequently in vitro transcribed using T7 RNA polymerase (Fermentas) in Transcription Buffer $(40 \mathrm{mM}$ Tris-HCl at $\mathrm{pH}$ 7.9, $6 \mathrm{mM} \mathrm{MgCl}_{2}, 10 \mathrm{mM}$ DTT, $10 \mathrm{mM} \mathrm{NaCl}, 2 \mathrm{mM}$ spermidine) and $2 \mathrm{mM}$ NTPs each. After $2 \mathrm{~h}$ of incubation at $37^{\circ} \mathrm{C}$, DNase I was added for $10 \mathrm{~min}$ followed by the addition of 1 volume of stop buffer $(80 \%[\mathrm{v} / \mathrm{v}]$ formamide, $50 \mathrm{mM}$ EDTA at $\mathrm{pH} 8.0,0.025 \%$ [w/v] bromphenolblue and $0.025 \%[\mathrm{w} / \mathrm{v}]$ xylene cyanole). Subsequently the products were purified on a $10 \%$ denaturing PAGE. Full-length products were excised and isolated by passive elusion.

Substrate strands $(50 \mathrm{pmol})$ of in trans ribozymes were radioactively labeled by adding $2 \mathrm{U}$ T4 PNK (Fermentas), $1 \mu \mathrm{Ci}$ ${ }^{32} \mathrm{P}-\boldsymbol{\gamma}$-ATP, and reaction buffer $(50 \mathrm{mM}$ Tris- $\mathrm{HCl}$ at $\mathrm{pH}$ 7.6; $25^{\circ} \mathrm{C}, 10 \mathrm{mM} \mathrm{MgCl}_{2}, 5 \mathrm{mM}$ DTT, $0.1 \mathrm{mM}$ spermidine and 0.1 $\mathrm{mM}$ EDTA). Reactions were incubated at $37^{\circ} \mathrm{C}$ for $30 \mathrm{~min}$, phenol/chloroform extracted and purified using a G25 column.

In trans HHR activities were determined by heating $200 \mathrm{nM}$ of ribozyme and $20 \mathrm{nM}$ of substrate strand in $50 \mathrm{mM}$ Tris- $\mathrm{HCl}$ at $\mathrm{pH}$ 7.5 to $95^{\circ} \mathrm{C}$ for $2 \mathrm{~min}$ followed by slowly cooling to $37^{\circ} \mathrm{C}$. Cleavage reactions were started by addition of varying concentrations of $\mathrm{Mg}^{2+}$. Reactions were quenched with stop buffer after defined time points. The reaction mix was separated by $10 \%$ PAGE. Visualization was performed using phosphorimaging. The data were fitted using following equation (Stage-Zimmermann and Uhlenbeck 1998):

$$
F_{t}=F-\left(F-F_{0}\right) * \mathrm{e}^{-\mathrm{kt}},
$$

with $F_{t}$ as the fraction cleaved at the time $t, F$ the maximal fraction cleaved, and $F_{0}$ the fraction cleaved before reaction was started.

\section{In cis $\mathrm{HHR}$ activities}

In cis cleaving HHRs were synthesized as described above, but with changed transcription conditions: instead of $2 \mathrm{mM}$ of each NTP, $0.5 \mathrm{mM}$ ATP, $2 \mathrm{mM}$ CTP, $2 \mathrm{mM} \mathrm{GTP}$, and $2 \mathrm{mM} \mathrm{UTP}, 1$ $\mu \mathrm{Ci}{ }^{32} \mathrm{P}-\alpha$-ATP was added. Furthermore, self-cleavage of the HHR in the transcription mix was inhibited by the presence of $50 \mu \mathrm{M}$ of a DNA blocking strand hybridizing to the catalytic core of the ribozyme corresponding to Salehi-Ashtiani and Szostak (2001).

In cis HHR activities were determined by heating $300 \mathrm{nM}$ of ribozyme with theophylline or caffeine in $50 \mathrm{mM}$ Tris- $\mathrm{HCl}$ at $\mathrm{pH}$ 7.5 to $95^{\circ} \mathrm{C}$ for $2 \mathrm{~min}$ and then slowly cooling down to $37^{\circ} \mathrm{C}$. Cleavage reaction was initiated by addition of $\mathrm{Mg}^{2+}$ to yield 0.2 $\mathrm{mM}$ final concentration.

\section{Plasmid constructions and in vivo screening}

HHR variants, "three-way theoHHAz-pool" and "cleaved fragment of three-way theoHHAz" were introduced into the pET16b_eGFP plasmid (kindly provided by the Scheffner laboratory, University of Konstanz) by performing a PCR with Phusion DNA Polymerase (Finzyme) using sequence-specific primers with the designed ribozyme sequences attached to the $5^{\prime}$ end of the primer (underlined).pET16b_eGFP was used as a template, except for three-way theoHHAz Pool construction pET16b_eGFP three-way HHR was used instead. The constructs and primers were as follows:

HHR: CGAAACGCGCTTCGGTGCGTCCTGGATTCCACGAAG GAGATATACCATGGG and TCCTATTTGGGACTCATCAGC TGGATGTACCGAAGGAGAAAGGGGAATTGTTATCCGCTC; Inactive HHR: CGAGACGCGCTTCGGTGCGTCCTGGATTCCAC GAAGGAGATATACCATGGG and TCCTATTTGGGACTCAT CAGCTGGATGTACCGAAGGAGAAAGGGGAATTGTTATCC GCTC;

Three-way HHR: CGCCGTAAGGCGTCCTGACTCCGAATGGA GTATCTATCCACGAAGGAGATATACCATGGG and TTTCG TCCTATTTGGGACTCATCAGCTGCTAGTACCGAAGGAGA AAGGGGAATTGTTATCCGCTC;

Inactive three-way HHR: CGCCGTAAGGCGTCCTGACTCCGA ATGGAGTATCTATCCACGAAGGAGATATACCATGGG and TCTCGTCCTATTTGGGACTCATCAGCTGCTAGTACCGAA GGAGAAAGGGGAATTGTTATCCGCTC;

Three-way theoHHAz pool: CCTTTCGGCTGGTATNNNNNCA GGACGCCTTACGGCGTTTCG and CCCTTGGCAGCGGAG TATCTATCCACGAAGGAGATATACC; and

Cleaved fragment: CCTTGGCAGCGGAGTATCTATCCACGAAG GAGATATACCATGGGC and GCCTTTCGGCTGGTATCGGA ACAGTATTTCTAGAGGGGAATTGTTATCC.

Prior to the PCR each primer was $5^{\prime}$ phosphorylated by T4 PNK (Fermentas). Subsequently to the PCR, the template plasmid was digested using the restriction enzyme DpnI. The PCR products containing the ribozyme sequences were blunt end ligated (Quick Ligase, New England BioLabs) and afterward transformed into E. coli BL21(DE3) gold (Stratagene). Single colonies were picked and grown in LB-Medium supplemented with $100 \mu \mathrm{g} \mathrm{mL}$ carbenecilline (Roth). To confirm successful cloning, the plasmids were isolated (Miniprep Kit, Qiagen) and sequenced. 
The three-way theoHHAz pool was constructed using primers randomized at the respective positions. Transformation was followed by isolation of single clones of the pool into 384 deepwell plates. For screening, eGFP expression in the absence and in the presence of $2 \mathrm{mM}$ theophylline was compared with out-grown cultures. E. coli were grown in LB-medium supplemented with $100 \mu \mathrm{g} \mathrm{mL}{ }^{-1}$ carbenecilline at $37^{\circ} \mathrm{C}$ until outgrown. A total of 100 $\mu \mathrm{L}$ of each culture were transferred into 96-well microplates and the fluorescence of the expressed eGFP (excitation wavelength $=$ $488 \mathrm{~nm}$, emission wavelength $=535 \mathrm{~nm}$ ) was determined using a TECAN M200 plate reader. For background subtraction an E. coli BL21(DE3) gold culture lacking the eGFP expression plasmid was treated equally.

\section{SUPPLEMENTAL MATERIAL}

Supplemental material can be found at http://www.rnajournal.org.

\section{ACKNOWLEDGMENTS}

J.S.H. gratefully acknowledges the VolkswagenStiftung for funding a Lichtenberg-Professorship. We thank Patrick Ketzer and Astrid Joachimi for excellent assistance.

Received June 13, 2008; accepted January 31, 2009.

\section{REFERENCES}

Amontov, S. and Jaschke, A. 2006. Controlling the rate of organic reactions: Rational design of allosteric Diels-Alderase ribozymes. Nucleic Acids Res. 34: 5032-5038.

Berens, C., Thain, A., and Schroeder, R. 2001. A tetracycline-binding RNA aptamer. Bioorg. Med. Chem. 9: 2549-2556.

Blount, K.F. and Uhlenbeck, O.C. 2002. The hammerhead ribozyme. Biochem. Soc. Trans. 30: 1119-1122.

Burke, D.H. and Greathouse, S.T. 2005. Low-magnesium, transcleavage activity by type III, tertiary stabilized hammerhead ribozymes with stem 1 discontinuities. BMC Biochem. 6: 14 .

Canny, M.D., Jucker, F.M., Kellogg, E., Khvorova, A., Jayasena, S.D., and Pardi, A. 2004. Fast cleavage kinetics of a natural hammerhead ribozyme. J. Am. Chem. Soc. 126: 10848-10849.

Carola, C. and Eckstein, F. 1999. Nucleic acid enzymes. Curr. Opin. Chem. Biol. 3: 274-283.

Cech, T.R. 2002. Ribozymes, the first 20 years. Biochem. Soc. Trans. 30: 1162-1166.

Collins, J.A., Irnov, I., Baker, S., and Winkler, W.C. 2007. Mechanism of mRNA destabilization by the glmS ribozyme. Genes \& Dev. 21: 3356-3368.

Fedor, M.J. and Westhof, E. 2002. Ribozymes: The first 20 years. Mol. Cell 10: 703-704.

Forster, A.C. and Symons, R.H. 1987. Self-cleavage of virusoid RNA is performed by the proposed 55-nucleotide active site. Cell 50: 9-16.

Furtig, B., Buck, J., Manoharan, V., Bermel, W., Jaschke, A., Wenter, P., Pitsch, S., and Schwalbe, H. 2007. Time-resolved NMR studies of RNA folding. Biopolymers 86: 360-383.

Hartig, J.S., Najafi-Shoushtari, S.H., Grune, I., Yan, A., Ellington, A.D., and Famulok, M. 2002. Protein-dependent ribozymes report molecular interactions in real time. Nat. Biotechnol. 20: 717-722.

Jenison, R.D., Gill, S.C., Pardi, A., and Polisky, B. 1994. High-resolution molecular discrimination by RNA. Science 263: 1425-1429.

Jose AM, Soukup GA, Breaker RR. 2001. Cooperative binding of effectors by an allosteric ribozyme. Nucl Acids Res. 29: 1631-1637.
Khvorova, A., Lescoute, A., Westhof, E., and Jayasena, S.D. 2003. Sequence elements outside the hammerhead ribozyme catalytic core enable intracellular activity. Nat. Struct. Biol. 10: 708-712.

Lescoute, A. and Westhof, E. 2006. Topology of three-way junctions in folded RNAs. RNA 12: 83-93.

Lynch, S.A., Desai, S.K., Sajja, H.K., and Gallivan, J.P. 2007. A highthroughput screen for synthetic riboswitches reveals mechanistic insights into their function. Chem. Biol. 14: 173-184.

Martick, M. and Scott, W.G. 2006. Tertiary contacts distant from the active site prime a ribozyme for catalysis. Cell 126: 309-320.

Micura, R. and Hobartner, C. 2003. On secondary structure rearrangements and equilibria of small RNAs. ChemBioChem 4: 984-990.

Ogawa, A. and Maeda, M. 2008. An artificial aptazyme-based riboswitch and its cascading system in E. coli. ChemBioChem 9: 206-209.

Piganeau, N., Jenne, A., Thuillier, V., and Famulok, M. 2001a. An allosteric ribozyme regulated by doxycyline. Angew. Chem. Int. Ed. Engl. 40: 3503.

Piganeau, N., Thuillier, V., and Famulok, M. 2001b. In vitro selection of allosteric ribozymes: Theory and experimental validation. $J$. Mol. Biol. 312: 1177-1190.

Salehi-Ashtiani, K. and Szostak, J.W. 2001. In vitro evolution suggests multiple origins for the hammerhead ribozyme. Nature 414: 82-84.

Soukup, G.A. and Breaker, R.R. 1999a. Design of allosteric hammerhead ribozymes activated by ligand-induced structure stabilization. Structure 7: 783-791.

Soukup, G.A. and Breaker, R.R. 1999b. Engineering precision RNA molecular switches. Proc. Natl. Acad. Sci. 96: 3584-3589.

Stage-Zimmermann, T.K. and Uhlenbeck, O.C. 1998. Hammerhead ribozyme kinetics. RNA 4: 875-889.

Suess, B. and Weigand, J.E. 2008. Engineered riboswitches-Overview, problems and trends. RNA Biol. 5: 24-29.

Tang, J. and Breaker, R.R. 1998. Mechanism for allosteric inhibition of an ATP-sensitive ribozyme. Nucleic Acids Res. 26: 4214-4221.

Uhlenbeck, O.C. 1987. A small catalytic oligoribonucleotide. Nature 328: $596-600$.

Weinberg, M.S. and Rossi, J.J. 2005. Comparative single-turnover kinetic analyses of trans-cleaving hammerhead ribozymes with naturally derived non-conserved sequence motifs. FEBS Lett. 579: 1619-1624.

Wieland, M. and Hartig, J.S. 2008. Improved aptazyme design and in vivo screening enable riboswitching in bacteria. Angew. Chem. Int. Ed. Engl. 47: 2604-2607.

Wieland, M., Benz, A., Klauser, B., and Hartig, J.S. 2009. Artificial ribozyme switches containing natural riboswitch aptamer domains. Angew. Chem. Int. Ed. Engl. [E-ub ahead of print]. doi: 10.1002.anie.200805311.

Win, M.N. and Smolke, C.D. 2007a. A modular and extensible RNAbased gene-regulatory platform for engineering cellular function. Proc. Natl. Acad. Sci. 104: 14283-14288.

Win, M.N. and Smolke, C.D. 2007b. RNA as a versatile and powerful platform for engineering genetic regulatory tools. Biotechnol. Genet. Eng. Rev. 24: 311-346.

Winkler, W.C. and Breaker, R.R. 2005. Regulation of bacterial gene expression by riboswitches. Annu. Rev. Microbiol. 59: 487517.

Winkler, W.C., Nahvi, A., Roth, A., Collins, J.A., and Breaker, R.R. 2004. Control of gene expression by a natural metabolite-responsive ribozyme. Nature 428: 281-286.

Yen, L., Svendsen, J., Lee, J.S., Gray, J.T., Magnier, M., Baba, T., D'Amato, R.J., and Mulligan, R.C. 2004. Exogenous control of mammalian gene expression through modulation of RNA selfcleavage. Nature 431: 471-476.

Yen, L., Magnier, M., Weissleder, R., Stockwell, B.R., and Mulligan, R.C. 2006. Identification of inhibitors of ribozyme self-cleavage in mammalian cells via high-throughput screening of chemical libraries. RNA 12: 797-806.

Zuker, M. 2003. Mfold web server for nucleic acid folding and hybridization prediction. Nucleic Acids Res. 31: 3406-3415. 

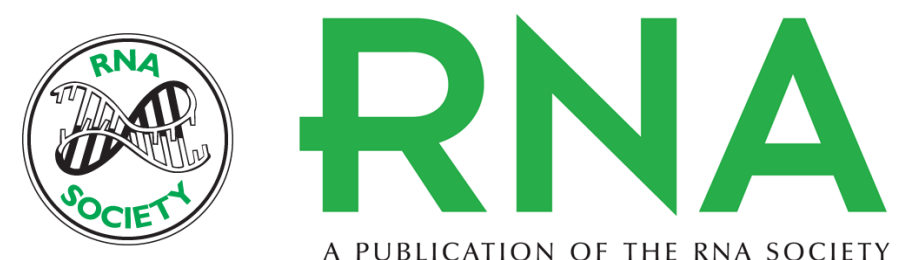

A PUBLICATION OF THE RNA SOCIETY

\title{
Expanded hammerhead ribozymes containing addressable three-way junctions
}

\author{
Markus Wieland, Manuela Gfell and Jörg S. Hartig
}

RNA 2009 15: 968-976 originally published online March 20, 2009

Access the most recent version at doi:10.1261/rna.1220309

\section{Supplemental http://rnajournal.cshlp.org/content/suppl/2009/03/23/rna.1220309.DC1 \\ Material}

References This article cites 39 articles, 6 of which can be accessed free at: http://rnajournal.cshlp.org/content/15/5/968.full.html\#ref-list-1

\section{License}
Email Alerting Receive free email alerts when new articles cite this article - sign up in the box at the Service top right corner of the article or click here.

